\section{La importancia del trabajo social y de cooperación altruista que desarrollan muchos cirujanos plásticos}

\section{Estimada Sra. Directora}

En primer lugar, me gustaría felicitar al Dr. Javier Fernández-Palacios por su artículo en el número 2 de 2020 de nuestra revista, Cirugía Plástica Ibero-Latinoamericana. ${ }^{(1)}$ Demuestra una vez más su gran experiencia en ayuda humanitaria quirúrgica. Y agradezco a la Dra. María del Mar Vaquero por reservar un espacio de esta publicación al trabajo social y de cooperación altruista que desarrollan muchos cirujanos plásticos.

He leído en esta sección interesantes artículos de nuestros colegas hispanoamericanos, que quizá nos llevan años de adelanto en esta labor. Es admirable cómo se organizan para tratar a un número enorme de pacientes. ${ }^{(2,3)}$ Especialmente en patología de fisurados. Pero también hace ya décadas que la Sociedad Española de Cirugía Plástica, nuestra SECPRE, destina esfuerzos a la ayuda humanitaria. Durante años se viajó a la misión de los Hermanos de San Juan de Dios de Chiclayo, en Perú. ${ }^{(4)}$

Por eso me gustaría traer a la memoria, añadiendo a lo que el Dr. Fernández-Palacios relata en su artículo, que ya en 2016 tuvo lugar el primer viaje humanitario en representación de la SECPRE a África. ${ }^{(5)}$ En aquellos años era vocal de relaciones institucionales de la SECPRE el Dr. Jesús Barón y llevaba entre manos iniciar una colaboración en Iquitos (Perú). Pero terminaba una terrible epidemia de ébola en Liberia y los Hermanos de San Juan de Dios solicitaron a la SECPRE que ese año se desplazase algún equipo de cirujanos plásticos al Hospital St. Joseph de Monrovia. Muchas ONGs habían dejado de ir esos años por allí. Cuando me llamó, conocía mi experiencia anterior de haber viajado varios años a Togo, en condiciones mucho más precarias a las que encontramos en Liberia y resultó un viaje inolvidable, que inició la "experiencia africana de la SECPRE".

África es un continente enorme, con realidades sociales muy diferentes y, como es lógico, nos referimos siempre a los países o regiones africanas pobres con recursos muy limitados y una estructura sanitaria muy precaria.

Me satisface haber sembrado la semilla del trabajo humanitario en mi propio Servicio de Cirugía Plástica, aquí en Alicante (España). La Dra. Maite Fernández me ha acompañado a Togo, a Liberia y después a Sierra Leona. Y varios miembros del Servicio han viajado después a Liberia y con seguridad lo retomarán los más jóvenes en cuanto estén formados y nos permitan viajar de nuevo. También en nuestra ciudad se puede cursar un máster oficial en cooperación al desarrollo, que oferta la Universidad Miguel Hernández cada dos años, por si fuese del interés del lector. ${ }^{(6)}$

Como cirujanos plásticos tenemos la fortuna de conocer unas técnicas quirúrgicas que, sin precisar grandes medios técnicos, pueden beneficiar a muchos pacientes y mejorar su calidad de vida futura. En nuestros viajes hemos llevado dermatomos, un mallador de piel y un doppler como instrumentos más sofisticados. Aunque estas misiones quirúrgicas sean cortas en el tiempo, a veces solo de un par de semanas, pueden ser muy eficaces si mantienen una continuidad anual o semestral. Se pueden resolver graves secuelas de quemaduras en varios tiempos quirúrgicos. Así conseguimos por ejemplo que Amadou, niño de 6 años pudiera extender sus dedos, hacer pinza e incluso llegar a escribir con una de sus manos, operándolo en dos campañas consecutivas.

Aún en situaciones difíciles como la actual pandemia Covid-19 que estamos sufriendo, tenemos que intentar mantener el espíritu de cooperación. No debemos ver el mundo desde nuestra burbuja privilegiada. El que haya programas diferentes de colaboración, como muestra esta sección de la revista, es bueno para todos y me gustaría instar a la SECPRE a que considere prioritario su programa de cooperación internacional dentro de su calendario anual de actividades. No se debería desperdiciar la experiencia ya adquirida ni el impulso de muchos cirujanos jóvenes. Esto contribuye también a la difusión hacia otras organizaciones internacionales de cooperación, que no piensan en cirujanos plásticos cuando solicitan cooperantes. Pueden descubrir a través de estos viajes la importancia de otro aspecto de nuestra especialidad, alejado de la cirugía puramente estética.

Por otro lado, estoy totalmente de acuerdo con el autor en el apoyo imprescindible de los sanitarios locales, enfermeros, misioneros o en el mejor de los casos, médicos o cirujanos de la región que se visita. Ellos conocen los casos, priorizan, dan continuidad a los cuidados postoperatorios de nuestros pacientes y de ese modo la campaña quirúrgica puede llegar a dar sus mejores frutos. 
De nuevo reitero mi enhorabuena al Dr. Fernández-Palacios, no sólo por su trabajo en múltiples viajes de cooperación, sino también por haber hecho el esfuerzo de revisar y ordenar su experiencia para divulgarlo.

Mi agradecimiento sincero

http://dx.doi.org/10.4321/S0376-78922021000100015

\author{
Elena LORDA BARRAGUER \\ Jefe de Servicio de Cirugía Plástica \\ Hosp. General Univ. De Alicante \\ Alicante, España \\ Correo electrónico: lorda_ele@gva.es
}

\section{Bibliografía}

1. Fernández-Palacios, J. et al. Requisitos, dinámica y ejecución de un proyecto de ayuda quirúrgica plástica en el continente africano: África Plástica. Cir. plást. iberolatinoam. 2020; 46(2):241-252.

2. Flores-Mendez MS., Medina-Flores GA. Jornadas humanitarias de labio y paladar hendidos en el sureste de la República Mexicana: 17 años de experiencia y 40 jornadas. Cir. plást. iberolatinoam. 2016;42(3): 399-405.

3. De la Cruz-Acosta F. Operación Sonrisa República Dominicana: 8 años de intensa y positiva experiencia. Cir. plást. iberolatinoam. 2016;42(1): 93-101

4. Barón.Thaidigsmann J. Campañas quirúrgicas humanitarias de la Sociedad Española de Cirugía Plástica, Reparadora y Estética en colaboración con los Hermanos de San Juan de Dios. Cir. plást. iberolatinoam. 2017; 43(4):425-437.

5. Lorda Barraguer E. La Sociedad Española de Cirugía Plástica viaja a África. Mi experiencia en Togo y Liberia. Cir. plást. iberolatinoam. 2017;43(2): 203-216.

6. Máster Interuniversitario en Cooperación al desarrollo.Universidad Miguel Hernández. Hthttp://mastercooperacion.edu. umh.es/

\section{Respuesta a la carta de la Dra. Elena Lorda Barraguer}

Le agradezco la oportunidad que me brinda de comentar la carta de la Dra. Elena Lorda, con la que comparto, aparte de inquietudes, una visión común de la cooperación plástica.

Dos formas distintas de ayuda humanitaria médica existen en la práctica. La primera es profesional, continua y con objetivos amplios y la desarrollan grandes ONGs como MSF o MDM con el apoyo de instituciones internacionales (ECHO, OCHA, ACNUR,...). La segunda es voluntaria, corta en el tiempo y generalmente recidivante y la realizan colectivos profesionales organizados en torno a Fundaciones o Instituciones Científicas o Sociales. Sin embargo, en situaciones de emergencia sanitaria la distinción no es tan sencilla, pues son las grandes organizaciones las encargadas de movilizar inmediatamente equipos de voluntarios quirúrgicos específicos con objetivos cortos y definidos.

El cirujano plástico encaja perfectamente tanto en las misiones cortas como en las situaciones de emergencia sanitaria: conflictos bélicos y terremotos, principalmente. La presencia de cirujanos plásticos en las emergencias es más difícil si no se tiene contacto con las grandes ONGs. Las instituciones oficiales también desconocen lo que podemos hacer sobre el terreno.

En 2016, la Agencia Española de Cooperación Internacional para el Desarrollo (AECID) creó el programa START (Spanish Technical Aid Response Team). A través de un registro de voluntarios hospitalarios de todos los perfiles, se creó un equipo de 48 profesionales para crear un hospital de campaña que se desplazaría de manera inmediata ante catástrofes humanitarias. Entre los perfiles requeridos figuraban todos los posibles, desde anestesistas, cirujanos generales y traumatólogos hasta nefrólogos. Sin embargo, lamentablemente no se solicitaron cirujanos plásticos.

Se comprueba una vez más que el desconocimiento sobre nuestra especialidad, desgraciadamente, trasciende lo más cercano para llegar a lo institucional. Y ahí es donde se deben centrar los esfuerzos no solo personales, sino institucionales. La SECPRE, como bien sugiere la Dra. Lorda, debería jugar un papel trascendental y proactivo en nuestra visualización tanto ante las ONGs como ante las Instituciones Gubernamentales.

Por otro lado, la ayuda humanitaria a África se ha ralentizado, más por la situación pandémica de Europa que por la de África.

Nuestra idea es reanudar desde que podamos las misiones quirúrgicas a África. El grupo de Alicante, liderado por Elena Lorda y con experiencia en países tan difíciles como Togo, Sierra Leona y Liberia, a buen seguro que espera impaciente para hacer lo mismo. Mi respeto para ellos y para todos los cirujanos plásticos que abandonan su burbuja de confort y seguridad para desarrollar la especialidad quirúrgica más bonita que hay sobre los pacientes que más necesitan de ella.

http://dx.doi.org/10.4321/S0376-78922021000100016

Javier FERNÁNDEZ-PALACIOS Jefe del Servicio de Cirugía Plástica Hospital Univ. de Gran Canaria "Dr. Negrín" Las Palmas de Gran Canaria Islas Canarias, España Correo electrónico: jferpalm@gmail.com 